\title{
Leches a base de plantas: Almendras ${ }^{1}$
}

\author{
Elena Torna, Daniela Rivero Mendoza y Wendy J. Dahl²
}

La leche de almendras, una alternativa a la leche de origen vegetal, se produce a partir de almendras y agua (Dhakal et al. 2014). La leche de almendras tiene su origen en la región mediterránea y se consume desde hace muchos años. En los Estados Unidos, hay varias marcas comercializadas de leche de almendras (Sethi et al. 2016). Esta publicación describe el perfil de nutrientes y los posibles beneficios y riesgos para la salud de la leche de almendras.

\section{¿Cómo se elabora la leche de almendras?}

Las marcas comerciales de leche de almendras están elaboradas a partir de agua filtrada y almendras, mezclas de vitaminas y minerales, sal y aditivos alimentarios, como gomas, ácido ascórbico, lecitina de girasol, entre otros (Silk n.d., Blue Diamond Almonds n.d.). También se pueden agregar edulcorantes y saborizantes.

La producción de leche de almendras comienza calentando el polvo de almendras en agua a $90^{\circ} \mathrm{C}$ (Sethi et al. 2016). Las partículas grandes se eliminan mediante centrifugación, un método de centrifugado / rotacion. La homogenización del líquido de almendras da como resultado una textura y apariencia uniforme, similar a la de la leche de vaca (Sethi et al. 2016). Los microorganismos se eliminan al tratar la leche de almendras a temperatura ultra alta (UHT) para producir un producto estable en almacenamiento (Sethi et al. 2016). Alternativamente, la leche de almendras se puede pasteurizar, lo que da como resultado un producto con una vida útil corta que necesita refrigeración (Sethi et al. 2016). Las altas temperaturas utilizadas con fines de seguridad alimentaria pueden provocar pérdidas de nutrientes (Sethi et al. 2016).

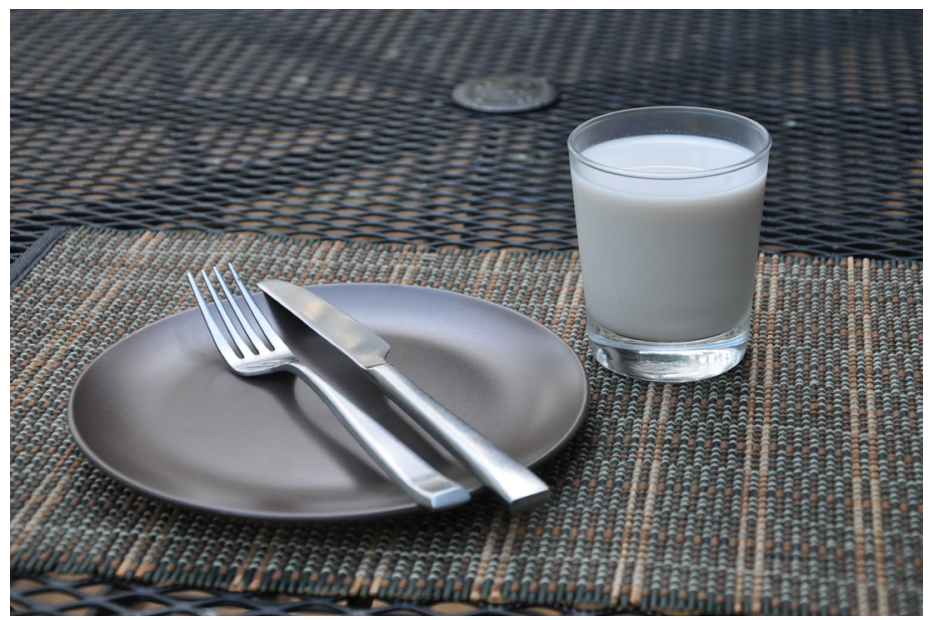

Figura 1. Crédito: Lincoln Zotarelli, UF/IFAS

\section{¿Cómo se compara el perfil de nutrientes de la leche de almendras con el de la leche de vaca?}

Las leches de origen vegetal varían en contenido de nutrientes. Las almendras son ricas en nutrientes proporcionanando ácidos grasos esenciales, vitaminas, fibra y fitoquímicos (USDA n.d.). Los minerales, como calcio, potasio, fósforo, magnesio, cobre, zinc y selenio, también se encuentran en

1. Este documento, FSHN20-48s, es uno de una serie de publicaciones del Ciencia de los Alimentos y Nutrición Humana, Servicio de Extensión Cooperativa de la Florida, Instituto de Alimentos y Ciencias Agrícolas, Universidad de la Florida. (UF/IFAS). Fecha de primera publicación: diciembre 2020. Visite nuestro sitio web EDIS en https://edis.ifas.ufl.edu.

2. Elena Torna, graduate student, Food Science and Human Nutrition Department; Daniela Rivero Mendoza, Extension and research coordinator; and Wendy J. Dahl, associate professor, Food Science and Human Nutrition Department; UF/IFAS Extension, Gainesville, FL 32611.

The Institute of Food and Agricultural Sciences (IFAS) is an Equal Opportunity Institution authorized to provide research, educational information and other services

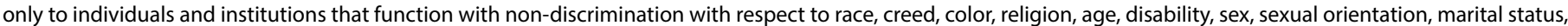

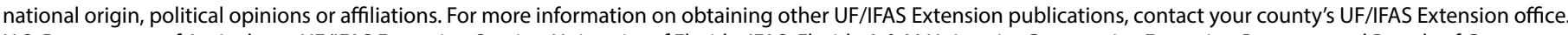
U.S. Department of Agriculture, UF/IFAS Extension Service, University of Florida, IFAS, Florida A \& M University Cooperative Extension Program, and Boards of County Commissioners Cooperating. Nick T. Place, dean for UF/IFAS Extension. 
las almendras (USDA n.d.). Las almendras también son una fuente de vitamina E. Sin embargo, la leche de almendras contiene muy pocas almendras por porción; por lo tanto, los niveles de estos nutrientes proporcionados son más bajos que una ración de almendras. La fortificación ayuda a asegurar una ingesta adecuada de vitaminas y minerales esenciales. La leche de almendras puede estar fortificada con calcio y vitaminas A y D.

La Tabla 1 resume las diferencias en los perfiles de nutrientes de la leche de almendras sin azúcar y fortificada y la leche de vaca; sin embargo, los perfiles de nutrientes de las leches de almendras saborizadas, endulzadas y sin fortificar difieren. Una porción de leche de almendras sin azúcar ( 1 taza) contiene aproximadamente 30 calorías, significativamente menos que las calorías de la leche de vaca. Sin embargo, una porción de leche de almendras tiene solo alrededor de $1 \mathrm{~g}$ de proteína, mientras que la leche de vaca contiene $8 \mathrm{~g}$. Una porción de leche de almendras tiene solo $2.5 \mathrm{~g}$ de grasa, similar a la cantidad de la leche de vaca baja en grasa. La leche de almendras no contiene grasas saturadas, al igual que la leche de vaca descremada. La leche entera, por otro lado, tiene 8 gramos de grasa, de los cuales aproximadamente 5 gramos son grasas saturadas. La leche de almendras no es una fuente de fibra dietética.

\section{¿Cuáles son los posibles beneficios para la salud de la leche de almendras?}

La leche de almendras puede ser una buena opción para los adultos alérgicos a la proteína de la leche de vaca o para quienes tienen intolerancia a la lactosa. La intolerancia a la lactosa es causada por la insuficiencia de lactasa, una enzima que digiere la lactosa (el azúcar de la leche de vaca). La leche de almendras no contiene lactosa y, por lo tanto, no causa síntomas en personas con mala digestión de lactosa (NLM n.d.). Sin embargo, las leches lácteas sin lactosa también están disponibles en el mercado y pueden ser apropiadas para las personas que intentan evitar la lactosa pero desean obtener los beneficios nutricionales de la leche de vaca.

La leche de almendras también puede ser un sustituto apropiado de la leche de vaca para veganos y adultos vegetarianos. Sin embargo, debe tenerse en cuenta que el perfil de nutrientes de la leche de almendras es bastante diferente al de la leche de vaca, ya que proporciona una cantidad significativamente menor de proteínas mientras proporciona ciertas vitaminas y calcio debido a la fortificación. Por último, la leche de almendras no contiene grasas saturadas, lo que puede resultar atractivo para las personas que intentan restringir su consumo de grasas saturadas. La leche de almendras sin azúcar y sin sabor suele ser baja en calorías, lo que puede ser apropiado para adultos que intentan perder peso.

\section{¿Cuáles son los posibles riesgos de consumir leche de almendras?}

En algunas poblaciones, como los adultos mayores, la sustitución de la leche de vaca por una alternativa a la leche de origen vegetal puede provocar una ingesta inadecuada de proteínas. Las nueces de árbol, incluidas las almendras, son una de las causas más comunes de reacciones alérgicas, las que algunas veces resultan fatales (McWilliam et al. 2015). Las almendras contienen un total de 188 proteínas y la amandina, o proteína principal de la almendra (AMP), se encuentra entre ellas. La amandina no se desnaturaliza después del tratamiento térmico y permanece intacta, lo que hace que la leche de almendras no sea adecuada para el consumo de personas con alergia a las nueces de árbol. La leche de almendras está etiquetada como un producto alimenticio que contiene alérgenos, como lo requiere la Ley de Protección al Consumidor y Etiquetado de Alérgenos Alimentarios (FALCPA) (FDA n.d.).

\section{¿Es la leche de almendras una opción adecuada para los niños?}

$\mathrm{Al}$ igual que otras leches de origen vegetal, el contenido de nutrientes de la leche de almendras no es equivalente al de la leche de vaca. La leche de almendras puede estar fortificada con vitaminas y calcio, que son esenciales para el desarrollo de huesos y dientes en niños y adolescentes (ODS n.d.). Sin embargo, la leche de almendras proporciona muy poca proteína para el crecimiento infantil (Silk n.d.). Las leches de origen vegetal con mucha menos proteína que la leche de vaca no deben utilizarse como reemplazo de los lácteos (Sethi et al. 2016). A los niños mayores de 2 años, que no pueden consumir leche de vaca, se les anima a beber alternativas a la leche vegetal fortificada que tengan al menos 6 gramos de proteína por porción, como la leche de soja (Sethi et al. 2016). La leche de almendras no debe administrarse a los bebés. Los bebés necesitan leche materna $y$, en ausencia de leche materna, una fórmula infantil adecuada.

\section{Resumen}

La leche de almendras es una alternativa a la leche popular. Si no está endulzada, proporciona significativamente menos calorías por porción que la leche de vaca, así como tampoco 
contiene grasas saturadas, lo que la hace útil para quienes restringen las calorías o la grasa. Si está fortificada, la leche de almendras proporciona niveles similares de calcio y vitaminas A y D a los de la leche de vaca y puede ser apropiada para las dietas de veganos y personas con alergia a la proteína de la leche o la soja. Sin embargo, los consumidores deben saber que, a diferencia de la leche de vaca, la leche de almendras contiene muy poca proteína, lo que la hace inapropiada como sustituto lácteo para los niños.

\section{Referencias}

Blue Diamond Almonds. n.d. "Unsweetened Original Almond Milk." Accessed September 1, 2020. https://www. bluediamond.com/brand/almond-breeze/almondmilk/ unsweetened-original

Dhakal, Santosh, Changqi Liu, Ying Zhang, Kenneth H. Roux, Shridhar K. Sathe, and V. M. Balasubramaniam. 2014. "Effect of High Pressure Processing on the Immunoreactivity of Almond Milk." Food Research International 62:2159-222. https://doi.org/10.1016/j.foodres.2014.02.021

McWilliam, Vicki, Jennifer Koplin, Caroline Lodge, Mimi Tang, Shyamali Dharmage, and Katrina Allen. 2015. "The Prevalence of Tree Nut Allergy: A Systematic Review." Current Allergy and Asthma Reports 15 (9): 54. https://doi. org/10.1007/s11882-015-0555-8

National Institute of Health Office of Dietary Supplements (ODS). n.d. "Calcium Fact Sheet for Health Professionals." Accessed September 1, 2020. https://ods.od.nih.gov/

factsheets/Calcium-HealthProfessional/

Sethi, Swati, Sanjeev K. Tyagi, and Rahul K. Anurag. 2016. "Plant-Based Milk Alternatives an Emerging Segment of Functional Beverages: A Review." Journal of Food Science and Technology 53 (9): 34089-3423. https://doi.org/10.1007/ s13197-016-2328-3

Silk. n.d. "Unsweet Almond Milk." Accessed September 1, 2020. https://silk.com/plant-based-products/almondmilk/ unsweet-almondmilk/

United States Department of Agriculture (USDA). n.d. "Fooddata Central." Accessed September 1, 2020. https:// fdc.nal.usda.gov/index.html

United States Food and Drug Administration (FDA). n.d. "Food Allergies: What You Need to Know." Accessed September 1, 2020. https://www.fda.gov/Food/ResourcesForYou/Consumers/ucm079311.htm
United States National Library of Medicine (NLM): Genetics Home Reference. n.d. "Lactose Intolerance." Accessed September 1, 2020. https://ghr.nlm.nih.gov/condition/ lactose-intolerance\#statistics 
Tabla 1. Perfil de nutrientes de la leche de almendras en comparación con la leche de vaca sin grasa, baja en grasa y entera.

\begin{tabular}{|c|c|c|c|c|}
\hline & $\begin{array}{l}\text { Leche de } \\
\text { almendras sin } \\
\text { azúcar ( } 1 \text { taza) }\end{array}$ & $\begin{array}{l}\text { Leche descremada } \\
\qquad(1 \text { taza) }\end{array}$ & $\begin{array}{l}\text { Leche semidescremada } \\
\text { (1 taza) }\end{array}$ & $\begin{array}{l}\text { Leche entera } \\
\qquad(1 \text { taza) }\end{array}$ \\
\hline Energía (calorías) & 30 & 83 & 102 & 150 \\
\hline Proteína (g) & 1 & 8 & 8 & 8 \\
\hline Grasa total (g) & 2.5 & 0 & 2.4 & 8 \\
\hline Grasa saturada (g) & 0 & 0.1 & 1.5 & 4.5 \\
\hline Carbohidrato (g) & 1 & 12 & 12 & 12 \\
\hline Fibra (g) & $<1$ & 0 & 0 & 0 \\
\hline Azúcar total (g) & 0 & 12 & 12 & 12 \\
\hline Vitamina A (mcg) & 150 & 149 & 142 & 112 \\
\hline Vitamina B12 (mcg) & NR & 1 & 1 & 1 \\
\hline Vitamina D (mcg) & 2.5 & 3 & 3 & 3 \\
\hline Calcio (mcg) & 450 & 298 & 305 & 276 \\
\hline Sodio (mg) & 125 & 102 & 107 & 105 \\
\hline Potasio (mg) & 170 & 381 & 366 & 322 \\
\hline
\end{tabular}

